\title{
EARLY YEARS OF THE EDMONTON BAR
}

\author{
L. D. HYNDMAN, Q.C.*
}

In preparation for the 75th anniversary celebration of the province, the Provincial Archives asked Mr. Hyndman to compile a record of the Edmonton legal community of 1905. To complement this he also researched the genesis of the five senior Edmonton firms.

A full discussion of the personalities involved in the development of the Edmonton legal community may be found in Mr. Hyndman's article, "The Early Years of the Edmonton Bar" published in the Alberta Law Review Twenty-fifth Anniversary Issue at page 30.

Subject to further research the partnership names and dates of the five senior firms over the years until about the First Great War appear to be probably:

1891 Beck \& McNamara

1893 Beck \& Emery

1902 Beck, Emery \& Dubuc

1904 Beck, Emery \& Newell

1905 Beck, Emery, Newell \& Bolton

1907 Emery, Newell \& Bolton

1910 Emery, Newell, Ford \& Bolton

1912 Emery, Newell, Ford, Bolton \& Mount

1916 Emery, Newell \& Ford

1919 Emery, Newell, Ford \& Lindsay

1888 Shaw \& Prince

1891 Bown \& Prince

1896 Bown \& Robertson

1903 Bown \& Dawson

1905 Bown, Dawson, Kennedy \& Hyndman

1906 Bown, Dawson, McDonald \& Hyndman

1907 Hyndman \& Hyndman

1911 Hyndman, Hyndman, Milner \& Matheson

1915 Hyndman, Milner \& Matheson

1919 Hyndman, Milner, Matheson, Carr \& Dafoe

1889 S. S. Taylor

1893 Taylor \& Taylor

1899 Taylor \& Boyle

1902 Taylor, Boyle \& Gariepy

1907 Taylor, Boyle \& Parlee

1912 Boyle, Parlee, Freeman, Abbott \& Mustard

1912 Parlee, Freeman \& Abbott

1917 Parlee, Freeman, Abbott \& McKay

1918 Parlee, Freeman, McKay \& Howson

1895 A. C. Rutherford

1899 Rutherford \& Jamieson

1910 Rutherford, Jamieson \& Grant Rutherford, Jamieson, Rutherford \& McCuaig Rutherford \& McCuaig

* Master in Chambers, Court of Queen's Bench of Alberta, Edmonton. 


\section{William Short \\ 1900 Short \& Cross \\ 1903 Short, Cross \& Biggar \\ 1904 Short, Cross, Biggar \& Ewing \\ 1910 Short, Cross, Biggar \& Cowan \\ 1912 Short, Cross \& Biggar \\ 1912 Short, Woods, Biggar \& Collison \\ 1913 Short, Cross, Biggar, Sherry \& Field \\ 1913 Short, Woods, Biggar \& Collison \\ 1919 Short \& Cross \\ 1919 Short, Cross, Maclean \& Macdonald}

Edmonton Bench and Bar Legal Directory-1905

BECK, N. D. D.

Beck, Enery \& Dubuc, Imperial Bank Bldg.

Phone No.

BIGGAR, O.M.

Short, Cross, Biggar \& Ewing, Merchants Bank Bldg.

BISHOP, E. T.

McLeod Block, Jasper Avenue

BOLTON, S. E.

Beck, Emery \& Dubuc, Imperial Bank Bldg.

BOWN, J. C. F.

Bown, Dawson, Kennedy \& Hyndman, Civic Bldg.

Taylor, Boyle \& Gariepy, Jasper Ave., Corner of McDougall Ave.

CROSS, C. W.

Short, Cross, Biggar \& Ewing, Merchants Bank Bldg.

DAWSON, H. J.

Bown, Dawson, Kennedy \& Hyndman, Civic Bldg.

DICKSON, S. A.

Robertson \& Dickson, Douglas Block, Jasper Ave. (and at Ft. Saskatchewan)

DUBUC, Lucien

Beck, Emery \& Dubuc, Imperial Bank Bldg.

EMERY, E. C.

Beck, Emery \& Dubuc, Imperial Bank Bldg.

191

EWING, A. F.

Short, Cross, Biggar \& Ewing, Merchants Bank Bldg.

GARIEPY, W.

Taylor, Boyle \& Gariepy, Jasper Ave., Corner of McDougall Ave.

GRIESBACH, W. A.

Griesbach \& O'Connor, National Trust Bldgs., Jasper Ave.

HYNDMAN, J. D.

Bown, Dawson, Kennedy \& Hyndman, Civic Bldg.

KENNEDY, J.F.

Bown, Dawson, Kennedy \& Hyndman, Civic Bldg.

LEMAISTRE, W.

Wallbridge \& LeMaistre, Sandison Block, Jasper Ave.

MacDONALD, J. K.

MacDonald \& MacKinnon, Union Bank Bldg., Jasper Ave.

MacKINNON, D. H.

MacDonald \& MacKinnon, Union Bank Bldg., Jasper Ave.

324 
MCNAMARA, D. J.

Attorney General's Dept. (Land Titles Office)

NEWELL, C. F.

Beck, Emery \& Dubuc, Imperial Bank Bldg.

O'CONNOR, G. B.

Griesbach \& O'Connor, National Trust Bldgs., Jasper Ave. 324

ROBERTSON, $\mathrm{H}$. H.

Robertson \& Dickson, Douglas Block, Jasper Ave.

(and at Ft. Saskatchewan)

SCOTT, David Lynch

Judge, Supreme Court, N.W.T.

SHORT, Wm.

Short, Cross, Biggar \& Ewing, Merchants Bank Bldg.

SIFTON, Arthur Lewis

Chief Justice, Superior Court, N.W.T.

TAYLOR, H. C.

Taylor, Boyle \& Gariepy, Jasper Ave., Corner of

McDougall Ave.

WALLBRIDGE, J. E.

Wallbridge \& LeMaistre, Sandison Block, Jasper Ave.

Strathcona

JAMIESON, F. C.

Rutherford \& Jamieson, Whyte Ave.

RUTHERFORD, A. C.

Rutherford \& Jamieson, Whyte Ave.

TIPTON, J. G.

Whyte Ave. W. of Main

\section{Addenda}

The foregoing "Legal Directory 1905" appears to be the "official" directory early in 1905 but there were a number of lawyers whose names were inadvertently omitted or who came here later in that year and consequently their names were not included in the earlier directory. So far as can be ascertained at present these "newcomers" probably were:

CORMACK, John

Noel, Noel \& Cormack

COWAN, I. S.

Police Magistrate

CRAWFORD, J. L.

DOWNES, G. F.

Strathcona

HARRISON, W. G.

LANDRY, H. L.

MACDONALD, J. K.

(Deputy Attorney General, Sept.-Dec. 1905)

de W. McDONALD, C.

NOEL, Auguste

Noel, Noel \& Cormack

NOEL, J. C.

Noel, Noel \& Cormack

WORSLEY, P.J. 